\title{
Diagnostic DNA-flow- vs. -image-cytometry in effusion cytology
}

\author{
Helma Motherby ${ }^{\mathrm{a}, *}$, Natalia Pomjanski ${ }^{\mathrm{a}}$, Mary Kube ${ }^{\mathrm{a}}$, Alexandra Boros ${ }^{\mathrm{a}}$, Thomas Heiden ${ }^{\mathrm{b}}$, \\ Bernhard Tribukait ${ }^{\mathrm{b}}$ and Alfred Böcking ${ }^{\mathrm{a}}$ \\ a Institute of Cytopathology, Heinrich-Heine-University, Moorenstr. 5, D-40225 Düsseldorf, Germany \\ ${ }^{\mathrm{b}}$ Department of Medical Radiobiology, Karolinska Institute, S-10401 Stockholm, Sweden
}

Received November 2000

Accepted April 2002

\begin{abstract}
Aims: To determine the sensitivity and specificity of flow- and image-cytometry for the detection of DNA-aneuploidy as a marker for malignant cells in effusions.

Methods: 200 effusions (80 tumor cell-positive, 74 negative and 46 cytologically equivocal) were stained with DAPI-SR for DNA-flow- and with Feulgen-Pararosaniline for -image-cytometry. They were measured using a PAS-flow-cytometer and an AutoCyte-QUIC-DNA-workstation according to the ESACP consensus reports for DNA-flow- and -image-cytometry, respectively [7,23,29,49].

Results: Sensitivity of DNA-aneuploidy for the identification of malignant cells was $32.1 \%$ for DNA-flow- and $75.0 \%$ for -image-cytometry, specificity of -euploidy in benign cells was $100.0 \%$ for both methods. Positive predictive value of DNAaneuploidy for the identification of malignant cells was $100.0 \%$ for both techniques, negative predictive value of DNA-euploidy was $48.6 \%$ for DNA-flow- and $72.0 \%$ for -image-cytometry.

Conclusions: Searching for DNA-aneuploidy as a diagnostic marker for neoplastic cells in serous effusions image-cytometry revealed superior sensitivity as compared with monoparametric flow cytometry.
\end{abstract}

Keywords: DNA-flow-cytometry, DNA-image-cytometry, serous effusion, cytopathology

\section{Introduction}

The sensitivity of conventional cytology for the detection of malignant cells in effusions is unsatisfactory, about $58 \%$; specificity is about $97 \%$. Improvement of diagnostic accuracy is therefore necessary in effusion cytology. Measurements identified DNA-aneuploidy in 95.4-100\% of cells in metastatic carcinomas and in $57.1-82.9 \%$ of cells in malignant mesotheliomas in malignant effusions. Our analyses achieved a specificity of $100 \%$ for the marker DNA-euploidy to confirm absence of malignant cells in effusions. Sensitivity for the detection of tumor cells in cytologically equivocal effusions was $55.9-82.9 \%$, respectively at a specificity of $94.1-94.7 \%$.

\footnotetext{
${ }^{*}$ Corresponding author: Prof. Dr. Alfred Böcking, Institute of Cytopathology, Heinrich-Heine-University Düsseldorf, Moorenstr. 5, D-40225 Düsseldorf, Germany. Tel.: +49 21181 18346; Fax: +49 21181 18402; E-mail: boecking@uni-duesseldorf.de.
}

The diagnostic value of DNA-cytometry for the identification of malignant cells in effusions has previously also been demonstrated by other authors. DNA-aneuploidy was detected in $49 \%$ of tumor cellpositive effusions by image cytometry [21] and in 21$81 \%$ by flow-cytometry $[16,18,19,30,32,34,35,37,51$, 56]. Three authors compared the prevalences of DNAaneuploidy in malignant effusions achieved by DNAflow- and image-cytometry respectively: $27 \%$ and $73 \%$ [54], 62\% and 77\% [1] and 65\% and $100 \%$ [39]. Yet unfortunately all these authors reported unsatisfactory specificities (Kapusta et al. [39] had 15\% false positives). The diagnostic value of DNA-cytometry, especially for the identification of cells from malignant mesotheliomas has also been demonstrated previously, but mostly on single-cell preparations from histological material by DNA-cytometry with prevalences of $48-53 \%[22,36,52]$. Only two authors dedicated their work to the cytometric identification of cells from malignant mesothelioma in effusions [17, 
25]. Both applied DNA-image-cytometry and achieved prevalences of DNA-aneuploidy of 59 and $89 \%$ in tumor cell-positive effusions.

DNA-cytometry has also been applied for the identification of malignant cells in cytologically equivocal effusions. Prevalences of DNA-aneuploidy in these were 12.5-60\%. Hedley et al. [30], Croonen et al. [14] and Joseph et al. [38] applied DNA-flow-cytometry, while Freni et al. [21], Fischler et al. [20] and MatterWalstra et al. [42] -image-cytometry. From these studies it may be concluded, that DNA-aneuploidy is a sufficiently sensitive and highly specific marker for the identification of malignant cells in cytologically equivocal effusions.

In this study we examined the effect of the following aspects on diagnostic accuracy of DNA-cytometry concerning the identification of aneuploid tumor cells: (1) improved precision of measurements [26,40], (2) new algorithms (or their combinations) for diagnostic data interpretation, (3) increased number of cells measured. Furthermore, the ability of both methods to distinguish between primary and secondary tumors of the serous membranes was investigated. Finally a comparison of the practicability and costs of both methods was made.

\section{Materials and methods}

\subsection{Specimens and patient population}

The subject of our study was 200 effusions of the serous cavities with cytologically tumorcell-positive (80), -negative (74) and equivocal (46) diagnoses. These consisted of pleural (142), peritoneal (48), pericardial (6) effusions and coul de sac specimens (4) routinely investigated between April and October 1998 in the Institute of Cytopathology.

\subsection{Staining of specimens}

For measurement of DNA-content by flowcytometry effusions were centrifuged at $340 \mathrm{~g} / 5 \mathrm{~min}$. After decanting the supernatant, the cell pellet was rinsed twice in phosphate buffered saline (PBS), resuspended, once again centrifuged and decanted. The pellets of hemorrhagic effusions were rinsed once in ammoniumchloride $(0.3 \%)$ and once in PBS. Following the procedures of Heiden et al. [31], the pellets were then fixed in $10 \%$ buffered formalin for about $16 \mathrm{~h}$. Centrifugation and decanting of the supernatant was repeated. The pellet was then fixed in $96 \%$ ethanol for $1 \mathrm{~h}$, followed by another centrifugation and decanting, rinsed in tap water for $20 \mathrm{~min}$ and again centrifuged. In order to separate cells and to dissolve the, cytoplasm $200 \mu \mathrm{l} 0.1 \%$ protease (Sigma, Deisenhofen, Germany, Nr.: P-8038, protease type XXXiV: bacterial) was added to the pellet for at least $1 / 2 \mathrm{~h}$ at $37^{\circ} \mathrm{C}$. The digestion was stopped by adding $1.5 \mathrm{ml}$ DAPISR (4'6-Diamidino-2-Phenylindole (Sigma, Deisenhofen, Germany, Nr.: D9542) and Sulforhodamine 101 (Sigma, Deisenhofen, Germany, Nr.: S7635). Before measurement the specimens were filtered through a nylon net (cell trics, $50 \mu \mathrm{m}$, Partec, Münster, Germany, Nr.: 06-4-2317).

Details of the procedure of cell preparation for DNA-image-cytometry were described in our previous papers $[43,46]$. In brief, for purposes of routine cytological diagnosis three slides were air-dried and stained according to May-Grünwald-Giemsa (MGG) (five further slides were immediately fixed in a modification of Delauney's solution and stained according to Papanicolaou for optional immunocytochemical staining [46, 47]).

\subsection{Cytological diagnosis}

The specimens were evaluated according to generally accepted diagnostic criteria $[2,3,41]$ described in our previous paper [45]. $80(40 \%)$ of the cases were diagnosed as tumorcell-positive, $74(37 \%)$ as -negative and $46(23 \%)$ as cytologically equivocal (doubtful or suspicious for malignancy) $[9,10]$.

\subsection{DNA-flow-cytometry}

The frequency peaks of the fluorescence of normal diploid reference cells (lymphocytes, mesothelial cells, macrophages and granulocytes), which were always contained in the effusions investigated, were set at channel 50. These were used as internal reference cells. The measurement was only accepted, if the coefficient of variation $(\mathrm{CV})$ of reference cells was $<6 \%$ (sigma $\mathrm{CV}=\mathrm{CV}$ based on the standard deviation) ([49] (half peak CV $\leqslant 3 \%)$ ).

Trout erythrocytes were used as an external standard (Partec, Münster, Germany, Nr. 06-5-7302. A correct adjustment resulted in narrow peaks $(\mathrm{CV} \sim 1 \%)$ with a doubling of modal values. The first peak was set at channel 25.

In the 80 malignant, 74 benign and 46 cytologically equivocal effusions a minimum of 20,000 cells 
were measured per specimen if present. This was the case in $81.5 \%$ (163/200) of effusions (in 105/200 cases the measurement was stopped at exactly 20,000 cells and in 58/200 cases more than 20,000 cells were measured, as only minor peaks in abnormal positions were seen in the histogram so that the measurement was continued). In four cases only $<5,000$ cells, in eight 5,001-10,000, in 17 cases 10,001-15,000 and in eight cases $15,001-19,999$ cells were measured, as routine specimens did not yield a higher number of cells. For measurements the PAS II (Particle Analysing System)-cytometer (Partec, Münster, Germany) was used. The performance of the system meets the requirements of the ESACP consensus report on standardization of DNA-flow-cytometry in clinical pathology [49]. The samples were analyzed as described by Heiden et al. [31]. DAPI was excited in the ultraviolet (350$400 \mathrm{~nm})$ and the fluorescence was measured in the blue region $(\sim 435 \mathrm{~nm})$. Usually a sample volume of $1.5 \mathrm{ml}$ or at least 20,000 nuclei were analyzed from each sample at a measuring rate of $\leqslant 100$ nuclei/sec. FSC listmode data storage occurred according to the recommendations published by the Data File Standards Committee of the Society of Analytical Cytology [15].

DNA-aneuploidy was assumed: if (1) the DNAindex of a frequency peak was $\langle 0.9\rangle$ or $\langle 1.1\rangle$ and $\langle 1.80\rangle$ or $\langle 2.20\rangle$ and $\langle 2.70\rangle$ or $\langle 3.30\rangle$ and $\langle 3.60\rangle$ or $\langle 4.40\rangle$ ("abnormal stemline"), which was determined by interactive marking its minimum and maximum in the histogram on the screen; or (2) if the stemline at a DNAindex of 2.0 showed a higher peak than that at index of 1.0 ("predominating tetraploid stemline"); or (3) if a double peak was seen. This was the case, if there was one "empty" channel between two peaks with a frequency value of $<75 \%$ of the lowest neighboring peak or two respectively three channels with a peak of $<80 \%$ or $<85 \%$, respectively.

\subsection{DNA-image-cytometry}

For DNA-measurement one previously MGGstained slide was later uncovered in xylene and subsequently Feulgen-stained in a temperature-controlled staining machine with Schiff's reagent according to the protocol applied in our previous papers $[5-7,13,43,46]$.

Measurements of nuclear DNA were performed as described in our previous papers [5-7,43,46]. In brief, 30 lymphocytes were measured as reference cells, meeting a coefficient of variation $\leqslant 5 \%$. The coefficient of correlation between nuclear area and integrated optical density (IOD) of reference cells was $r<0.4$ [29].
Subsequently, if present, 300 atypical or abnormal cells were measured per specimen interactively at random. This was the case in $59.5 \%(119 / 200)$ of the cases. The measurement was stopped at 300 cells in 34 cases and in further 85 cases it was continued measuring $>300$ cells. Otherwise, only the available cells were measured: four cases revealed $<50$ cells; 10 cases 51-100 cells; 24 cases $101-150 ; 13$ cases 151-200; 21 cases 201-250 and nine cases 251-299 cells, as routine material did not yield a higher number of cells. The AutoCyte QUIC-DNA-Workstation (AutoCyte Inc., Burlington, NC, USA) was used for the measurements. The performance of the system meets the requirements of the updated consensus report of the ESACP task force on standardization of diagnostic DNA-image-cytometry [7,29]. The data were diagnostically interpreted as described in our previous paper [43]. DNA-aneuploidy was assumed if (1) an abnormal DNA-stemline (STL) was detected (DNAindex $\langle 0.90\rangle$ or $\langle 1.10\rangle$ and $\langle 1.80\rangle$ or $\langle 2.20\rangle$ and $\langle 3.60\rangle$ and $\langle 4.40\rangle[6,7,28,29,48]$, and/or (2) the coefficient of variation $(\mathrm{CV})$ of the first DNA-stemline was $>10 \%$, and/or (3) cells $>9 \mathrm{c}$ occurred (9c exceeding events $(9 \mathrm{c} \mathrm{EE}))$ [12].

\subsection{Feasibility}

One of the aims of this study was the comparison of the two methods with reference to the feasibility of measuring cytologically tumorcell-negative, -positive and equivocal effusions taking into account the total number of measurable cells as well as the distribution of different cell types (ratio of tumorcells to non-tumorcells (lymphocytes, mesothelial cells, macrophages and granulocytes)). The different cell types were analyzed by immunocytochemical staining (for details of the procedure [46,47]) of the identical slides and microscopical counting using an ocular grid with $10 \times 10$ squares.

\subsection{Validation of cytological diagnoses}

According to patient follow-up the investigated effusions of the serous membranes were classified as either containing malignant cells or not. We accepted patient histories as presenting sufficient evidence for the presence or absence of tumor cells in effusions. These revealed either histologic follow-up of the serous membranes themselves $(34 / 200=17.0 \%)$ or of the respective disease (primary tumor or benign disease, for example biopsy of the liver in cirrhosis of the liver) 
$(81 / 200=40.5 \%)$. Clinical evidence for a malignant nature of the effusion was considered valid, applying such diagnostic techniques as radiology and computer tomography $(85 / 200=42.5 \%)$. Patients presenting abnormal cells in effusions revealed the following primary tumors: carcinomas of the breast (14), the ovary (12), the parotid gland (1), the lung (25), the esophagus (1), the stomach (12), the colon (9), the gallbladder (1), the pancreas (2), the liver (1), the kidney (2), the urinary bladder (2) as well as carcinomas of unknown primary (8). Furthermore, malignant mesotheliomas (9) and leukemias/lymphomas (9) occurred. Non-malignant cases showed the following basic diseases: pneumonia/pleuritis (20), congestive heart failure (25), pneumothorax (2), rheumatic polyarthritisserositis (2), radiation with generalized edema (2), renal insufficiency (2), cirrhosis of the liver (14), hepatitis (1), pancreatitis (1), peritonitis (1), endometriosis (1), rupture of an ovarian cyst (1), postoperative trauma (7) and lymphohistiocytosis (1). Amongst the tumor cell-negative effusions there were 12 cytologically false negative due to carcinoma of the lung (3), the parotid gland (1), the ovary (2), the cervix (1), leukemias/lymphomas (3) and sarcomas of the stomach (2).

\section{Results}

\subsection{Tumorcell-negative effusions of the serous membranes}

None of the DNA-histograms of mesothelial and inflammatory cells in non-malignant, inflammatory or reactive effusions revealed any of the above mentioned criteria of DNA-aneuploidy, neither by DNAflow- nor -image-cytometry and were therefore consequently all interpreted as DNA-euploid. This corresponds to a specificity of both DNA-cytometric methods of $100.0 \%$ to detect benignity in normal or reactive mesothelial cells. In DNA-flow-cytometry the DNAindices were close to channel 50 (mean 49.7; range 45.0-52.0), on which the fluorescence intensity of normal diploid reference cells had been fixed previously. In DNA-image-cytometry the modal values were close to $2 \mathrm{c}$ (mean 2.02c; range 1.84-2.18c). The mean $\mathrm{CVs}$ of the first DNA-stemlines were $3.70 \%$ (sigma-CV) (range 1.88-6.68\%) in DNA-flow- and $2.04 \%$ (range $0.67-4.92 \%$ ) in -image-cytometry.
Table 1

Prevalence of DNA-aneuploidy in effusions of primary and secondary tumors serous of the membranes (DNA-flow- and -imagecytometry)

\begin{tabular}{lcc}
\hline DNA-ploidy-status & \multicolumn{2}{c}{ Tumor cell-positive effusions } \\
& \multicolumn{2}{c}{$n=80(100.0 \%)$} \\
\cline { 2 - 3 } & DNA-flow-cytometry & DNA-image-cytometry \\
\hline DNA-non-aneuploid & $33(41.3 \%)$ & $10(12.5 \%)$ \\
DNA-aneuploid & $47(58.8 \%)$ & $70(87.5 \%)$ \\
\hline
\end{tabular}

\subsection{Tumor cell-positive effusions of the serous membranes}

The DNA-histograms of metastatic carcinomas and malignant mesotheliomas of the serous membranes showed, according to the above mentioned algorithms of either DNA-cytometric method, none, one, two or all aspects of DNA-aneuploidy. On this basis, 47/80 effusions measured by DNA-flow- and 70/80 by -imagecytometry were DNA-aneuploid (Table 1). This corresponds to a prevalence of DNA-aneuploidy in malignant cells in effusions of $58.8 \%$ and $87.5 \%$, respectively (metastatic carcinomas: $60.0 \%$ and $92.9 \%$, respectively; mesotheliomas: $50.0 \%$ and $66.7 \%$, respectively; lymphomas: $50.0 \%$ and $25.0 \%$, respectively). Table 2 shows the frequency of occurrence of different aspects of DNA-aneuploidy in effusions due to metastatic carcinomas and mesotheliomas of the serous membranes. It clearly demonstrates that an abnormal stemline in DNA-flow- and 9cEE in -imagecytometry were the most frequent aspects of DNAaneuploidy and that combined application of different algorithms increased the rate of its detection.

It is obvious that in tumor cell-positive effusions all of the above mentioned aspects of DNA-aneuploidy, with differing sensitivities, are often well enough represented to serve as sensitive criteria for the identification of aneuploidy. One abnormal stemline was observed in DNA-flow-cytometry in $18.8 \%$ and in DNA-image-cytometry in $26.3 \%$ of the malignant tumors of the serous membranes. Two abnormal stemlines were found in $36.6 \%$ and in $42.5 \%$, respectively and multiple DNA-stemlines in $3.8 \%$ and $6.3 \%$ of cases. Apart from abnormal stemlines (53.8\%) in DNA-flow-cytometry a predominant tetraploid stemline $(6.3 \%)$ was the second most frequent aspect of DNA-aneuploidy. A double peak served as a criterion of DNA-aneuploidy in 3.8\% of the tumors of the serous membranes (Table 2). In DNA-image-cytometry, next to $9 \mathrm{cEE}(77.5 \%)$, abnormal stemlines were the second most frequent aspect of DNA-aneuploidy (75.0\%). An 
Table 2

Prevalence of different aspects of DNA-aneuploidy in effusions of primary and secondary tumors of the serous membranes (DNA-flowand -image-cytometry) (STL $=$ stemline; $\mathrm{CV}=$ coefficient of variation; $9 \mathrm{cEE}=9 \mathrm{c}$ exceeding events)

\begin{tabular}{lc}
\hline Aspects of DNA-aneuploidy & $n=80(100.0 \%)$ \\
\hline DNA-flow-cytometry & $43(53.8 \%)$ \\
Abnormal STL & 15 \\
One & 29 \\
Two & 3 \\
Multiple & $5(6.3 \%)$ \\
Stemline 4c $>$ 2c & $3(3.8 \%)$ \\
Double peak & \\
DNA-image-cytometry & $60(75.0 \%)$ \\
Abnormal STL & 21 \\
One & 34 \\
Two & 5 \\
Multiple & $19(23.8 \%)$ \\
CV of first STL $\geqslant 10 \%$ & $62(77.5 \%)$ \\
9cEE &
\end{tabular}

Table 3

Prevalence of DNA-aneuploidy in cells of cytologically equivocal effusions

\begin{tabular}{lcc}
\hline DNA-ploidy-status & \multicolumn{2}{c}{$\begin{array}{c}\text { Cytologically equivocal effusions } \\
n=46(100.0 \%)\end{array}$} \\
\cline { 2 - 3 } & DNA-flow-cytometry & DNA-image-cytometry \\
\hline DNA-non-aneuploid & $37(80.4 \%)$ & $25(54.3 \%)$ \\
DNA-aneuploid & $9(19.6 \%)$ & $21(45.7 \%)$ \\
\hline
\end{tabular}

abnormally high $\mathrm{CV}$ of the first stemline served as a criterion of DNA-aneuploidy in $23.8 \%$ (Table 2).

Whereas in $53.8 \%$ of malignant effusions in DNAflow- and in $75.0 \%$ in -image-cytometry DNA-aneuploidy was identified by an abnormal stemline alone, the identification rate increased to $55.0 \%$ if a predominant tetraploid stemline and to $58.8 \%$ if additionally a double peak was used as a criterion of DNAaneuploidy in DNA-flow-cytometry. In DNA-imagecytometry the identification rate increased to $87.5 \%$, if additionally 9cEE was used as a criterion of DNAaneuploidy. An abnormally high CV of the first stemline as a criterion did not further increase the identification rate (Table 3 ).

Amongst the 80 tumor cell-positive effusions in this study there were only six mesotheliomas, of which three were DNA-aneuploid by DNA-flow- and four by -image-cytometry. In all of these the DNA-histogram of flow-cytometry showed a peridiploid stemline, in two of the aneuploid effusions additionally a hypodiploid and in one a hypotetraploid stemline was ob-
Table 4

Prevalence of different aspects of DNA-aneuploidy in cytologically equivocal effusions (DNA-flow- and -image-cytometry) (STL $=$ stemline $\mathrm{CV}=$ coefficient of variation; $9 \mathrm{cEE}=9 \mathrm{c}$ exceeding events)

\begin{tabular}{lc}
\hline Aspects of DNA-aneuploidy & $n=46(100.0 \%)$ \\
\hline DNA-flow-cytometry & $9(19.6 \%)$ \\
Abnormal STL & 5 \\
One & 4 \\
Two & - \\
Multiple & - \\
Stemline 4c $>$ 2c & - \\
Double peak & \multicolumn{2}{c}{-} \\
DNA-image-cytometry & $15(32.6 \%)$ \\
Abnormal STL & 10 \\
One & 5 \\
Two & - \\
Multiple & $3(6.5 \%)$ \\
CV of first STL $\geqslant 10 \%$ & $14(30.4 \%)$ \\
9cEE &
\end{tabular}

served. In image-cytometry there was no specific pattern to be seen in the histograms.

\subsection{Diagnostic accuracy in cytologically equivocal effusions of the serous cavities}

9/46 and 21/46 of cytologically equivocal effusions were DNA-aneuploid in DNA-flow- and -imagecytometry, respectively (Table 3). This corresponds to a prevalence of DNA-aneuploidy in cytologically equivocal effusions of $19.6 \%$ and $45.7 \%$, respectively. In cytologically equivocal effusions an abnormal stemline was the most frequent aspect of DNA-aneuploidy using DNA-flow-cytometry (19.6\% (9/46)). The combined application of three different algorithms did not increase the rate of detection (Table 4). In DNA-imagecytometry an abnormal stemline was the most frequent aspect of DNA-aneuploidy (32.6\% (15/46)), followed by 9 cEE $(30.4 \%(14 / 46))$ and an abnormally high CV of the first stemline (6.5\% (3/46)). The combined application of different algorithms increases the detection rate of DNA-aneuploidy (Table 4).

It is obvious, that all aspects of DNA-aneuploidy, with differing sensitivities, are often enough represented to serve as a sensitive criterion for identification in cytologically inconclusive effusions as well as in effusions suspicious for malignancy. One abnormal stemline was observed in $10.9 \%$ (5/46) in DNA-flowand in $21.7 \%$ (10/46) of the cases in -image-cytometry, respectively. Two abnormal stemlines were found in 
Table 5

DNA-flow- and -image-cytometry in equivocal effusions

\begin{tabular}{lcc}
\hline & $\begin{array}{c}\text { DNA-flow-cytometry } \\
\text { (DNA-aneuploidy) }\end{array}$ & $\begin{array}{c}\text { DNA-image-cytometry } \\
\text { (DNA-aneuploidy) }\end{array}$ \\
\hline Prevalence & $19.6 \%$ & $45.7 \%$ \\
Sensitivity & $(9 / 46)$ & $(21 / 46)$ \\
& $32.1 \%$ & $75.0 \%$ \\
Specificity & $(9 / 28)$ & $(21 / 28)$ \\
& $100.0 \%$ & $100.0 \%$ \\
Positive & $(18 / 18)$ & $(18 / 18)$ \\
predictive value & $100.0 \%$ & $100.0 \%$ \\
Negative & $(9 / 9)$ & $(21 / 21)$ \\
predictive value & $48.6 \%$ & $72.0 \%$ \\
\hline
\end{tabular}

$8.7 \%(4 / 46)$ and in $10.9 \%(5 / 46)$, respectively; multiple stemlines were not found. A double peak or a predominant tetraploid stemline as criterion for DNAaneuploidy were not found in cytologically equivocal effusions. Using flow-cytometry $9 \mathrm{cEE}$ served as criterion for DNA-aneuploidy in 30.4\% (14/46) and an abnormally high $\mathrm{CV}$ of the first stemline in $6.5 \%(3 / 46)$ in DNA-image-cytometry (Table 4).

In this study three cytologically equivocal effusions were due to malignant mesotheliomas. DNAaneuploidy was detected in two of these by DNA-flowand by -image-cytometry each by detection of an abnormal steamline by either method.

The positive predictive value of the marker DNAaneuploidy by DNA-flow- as well as -image-cytometry was $100.0 \%$ in cytologically equivocal effusions (Table 5). The negative predictive value of DNA-nonaneuploidy was $48.6 \%$ and $72.0 \%$, respectively, as only 18 of 37 and 18 of 25 non-aneuploid cases, respectively showed benign histories. Sensitivity for identification of malignancy in equivocal effusions was $32.1 \%$ by DNA-flow- and $75.0 \%$ by -image-cytometry, specificity of DNA-non-aneuploidy for benignity was $100.0 \%$ by both methods. Considering the achieved prevalences of DNA-aneuploidy in cytologically positive $(n=80)$ and negative $(n=74)$ as well as equivocal ( $n=46$ ) effusions, the total positive predictive value of DNA-aneuploidy was $100.0 \%$ by both DNA-flow- and -image-cytometry, the total negative ones were $63.9 \%$ and $84.4 \%$, respectively. Total sensitivity was $51.9 \%$ and $84.3 \%$, respectively, total specificity $100.0 \%$ for both methods. Total diagnostic accuracy was $74.0 \%$ and $91.5 \%$, respectively.

\subsection{Feasibility}

The feasibility of both DNA-cytometrical methods was compared. In 160/200 effusions both methods achieved identical results in the detection of non-aneuploidy or aneuploidy in tumorcell-negative, -positive and cytologically equivocal effusions respectively. In 40/200 cases of tumorcell-containing effusions (26 cytologically tumor cell-positive and 14 cytologically equivocal effusions) image-cytometry detected aneuploidy while flow-cytometry did not. $27.5 \%$ (11/40) of these effusions showed an absolute percentage of tumor cells less than $5 \%(7.5 \%(3 / 40)<1 \%$, $12.5 \%(5 / 40) 1-2.5 \%$ and $7.5 \%$ (3/40) $2.5-5 \%)$. Another $27.5 \%(11 / 40)$ revealed a high percentage of tumorcells (11.8-99.0\% (mean 43.7\%)), but not consequently with the same DNA-content and therefore not all represented in one peak, but instead distributed all over the DNA-histogram. Furthermore, in 27.5\% (11/40) single cells with a high DNA-content were not detected, in $20.0 \%(8 / 40)$ of these this was the only criterion for aneuploidy in image-cytometry (in $17.5 \%$ (7/40) one cell and $2.5 \%$ (1/40) three cells with a DNAcontent $>9 \mathrm{c}$ were detected). In $10 \%$ (4/40) small double peaks were not detected. In $12.5 \%$ (5/40) the absolute number of measurable cells within the effusions was too small $(<20,000)$ and the CV was too high.

\section{Discussion}

Improvement of the sensitivity for the detection of tumor cells in effusions without loss of specificity is desirable. DNA-cytometry is a promising adjuvant method for this purpose. This study compares the diagnostic accuracy of DNA-flow- vs. -image-cytometry in 200 effusions applying different algorithms for the identification of DNA-aneuploidy using instruments and protocols which promise high precision of measurement.

Image-cytometric analysis of primary and secondary tumors of the serous membranes showed DNA-aneuploidy in $87.5 \%$, -flow-cytometrical investigation in only $58.8 \%$ (Table 1). Specificity of both methods was $100.0 \%$.

The detection rate in tumorcell-positive effusions increased by additional application of up to three algorithms. If a high detection rate of DNA-aneuploidy is to be achieved, we recommend using more than one abnormal aspect of the DNA-distribution. 
The sensitivity of DNA-cytometry for the identification of malignancy in cytologically equivocal effusions was $32.1 \%$ for DNA-flow- and $75.0 \%$ for -imagecytometry. Specificity of DNA-non-aneuploidy for the identification of non-neoplastic cells was $100.0 \%$ for both methods (Table 5). The positive predictive value of DNA-aneuploidy in cytologically equivocal effusions was $100.0 \%$ for both methods. The negative predictive value of DNA-non-aneuploidy was $48.6 \%$ for DNA-flow- and $72.0 \%$ for -image-cytometry.

A proliferating cell population with an abnormal DNA-content is supposed to represent cells with chromosomal aneuploidies and this is often denominated as an aneuploid DNA-stemline. Whereas cells belonging to a DNA-stemline are supposed to be cytogenetically identical proliferating cells, rare events with DNA-contents $>9 \mathrm{c}$ most likely represent nonproliferating cells with different chromosomal aneuploidies and abnormally high numbers of chromosomes. The latter cells can only be identified with DNA-image-cytometry including a visual control for diagnostic purposes.

The different prevalences of DNA-aneuploidy found in other studies on malignant effusions may amongst other factors be due to the number and type of algorithms applied for the interpretation of histograms.

Most authors have used flow-cytometry to detect tumor cells in effusions. Yet the sole algorithm applied in most studies was the detection of an abnormal or aneuploid DNA-stemline. Only Stonesifer et al. [56] demonstrated the possibility of increasing the detection rate of DNA-aneuploidy by the analysis of different abnormal aspects of the histograms. They considered the occurrence of $>2.5 \%$ of the population in the G2/M-phase of the cell cycle, a wide G0/1-phase-peak as well as an asymmetry or a shoulder of an G1-peak as a further aspect of DNA-aneuploidy, by which they achieved a sensitivity of $88 \%$ of all effusions with three false positive cases.

Several authors have applied DNA-image-cytometry. Although Kapusta et al. [39] achieved a prevalence of $100 \%$, their specificity was low (15\% DNAcytometrically false positives). The sole algorithm applied in most of these studies was the detection of an abnormal or aneuploid stemline, and by Freni et al. [21] the observance of DNA-values $>8 \mathrm{c}$. In our opinion, this approach is not sufficient to achieve an adequately high detection rate of DNA-aneuploidy. As demonstrated in previous studies $[43,44]$ we, have suggested the application of more than one algorithm for the detection of DNA-aneuploidy. Fischler et al. [20] showed the possibility of increasing the detection rate of DNA-aneuploidy by analysis of different aspects of the histograms. They considered the occurrence of multiple DNA-stemlines and nuclear values $>5 \mathrm{c}$.

We recommend the application of the following algorithms. For DNA-flow-cytometry: the occurrence of an abnormal stemline, a predominant tetraploid stemline or a double peak. For DNA-image-cytometry we propose the occurrence of an abnormal stemline and DNA-values $>9 \mathrm{c}$.

\subsection{Algorithms for the detection of DNA-aneuploidy by flow-cytometry}

DNA-aneuploidy is usually assumed, if the modal value of the G0/1-fraction of the cell population differs more than $10 \%$ (or recently only $5 \%$ ) of that of the diploid reference cell population or one of its integer valued multiples [49]. We believe, that even smaller deviations may be detected (occurrence of a "double peak"). As soon as chromosomal aberrations lead to a change of DNA-content, which differs statistically highly significantly from that of normal, nonneoplastic cells, DNA-aneuploidy may be assumed [8]. In our opinion, the prevalence of DNA-aneuploidy in tumor cell-positive effusions may be increased by a higher precision of DNA-measurements, for example, by use of the PAS-Flowcytometer (Partec, Münster, Germany) applied in this study, the use of DAPI as a DNA-specific fluorochrome instead of propidiumiodide and the simultaneous application of three different algorithms for the detection of DNA-aneuploidy. An abnormal DNA-stemline was the most frequent aspect of aneuploidy in effusions containing tumor cells. As the relation of the modal values of the DAPIfluorescence of analysis- and reference cells may differ slightly as a result of differences in fixation and staining, an empirically found range must be considered. As, in our study, this ranged from channel 45.0-52.0 for lymphocytes, granulocytes, mesothelial cells or macrophages, we consequently decided on threshold values of DNA-indices $\langle 0.90\rangle 1.10$, in order to assume DNA-stemline-aneuploidy. This corresponds well with the range of $\pm 5 \%$ of the modal values of normal diploid cells mentioned by Ormerod et al. [49]. A further algorithm for the detection of DNA-aneuploidy, applied by us, is the occurrence of a predominant tetraploid stemline, which contains more cells than the respective diploid one. Furthermore, double peaks served as a criterion for the assumption of DNA-aneuploidy in effusions of the serous mem- 
branes. In 40 cases of tumor cell-containing effusions (26 cytologically tumor cell-positive and 14 cytologically equivocal effusions) image-cytometry detected aneuploidy while flow-cytometry did not. The main reason was the overlap of a small population of tumor cells by a great number of normal cells (lymphocytes, mesothelial cells, macrophages, granulocytes). This was due to the following facts (more than one cause possible): $27.5 \%$ of these effusions showed an absolute percentage of tumor cells less than 5\%. Another $27.5 \%$ revealed a high percentage of tumorcells, but these did not all have the same DNA-content and consequently were not all represented in one peak, but instead distributed all over the DNA-histogram. Furthermore, in $27.5 \%$ single cells with a high DNA-content were not detected because of overlapping of normal cells by doublets, triplets and quadruplets. These problems can be solved by including specific immunologic staining with epithelial antibodies and two wavelength double parameter measurements. In $10 \%$ small double peaks were not detected because of insufficient precision of measurement. In $12.5 \%$ the absolute number of measurable cells within the effusions was too small $(<20,000)$ and the CV was too high. This reflects the situation of routine effusion material.

\subsection{Algorithms for the detection of DNA-aneuploidy by image-cytometry}

We assume that the high prevalence of DNA-aneuploidy in tumor cell-positive effusions seen by us may be due to the simultaneous application of two different algorithms for the detection of DNA-aneuploidy. We used threshold values of $<1.80 \mathrm{c}$ and $>2.20 \mathrm{c}$ to assume DNA-stemline-aneuploidy. A further frequently applied marker for DNA-aneuploidy is the occurrence of single cells with a DNA-content over certain threshold values in cells. In tissues with euploid polyploidisation up to tetraploid cell populations, as the urothelium or mesothelium, only the occurrence of cells with a DNAcontent $>9 c$ ( $9 c$ exceeding events) may be considered as a marker for single-cell-DNA-aneuploidy $[4,12]$, as no cells of reactive, proliferating or benign mesothelium will exceed these values. In this aspect, we do not agree with other authors $[20,50]$, who already consider the occurrence of cells with values $>5 \mathrm{c}$ as a marker for DNA-aneuploidy in cells with euploid polyploidisation. The coefficient of variation of the DNA-stemline depends on the precision of DNA-measurements, the rate of proliferation and the occurrence of aneuploid nuclear DNA-values. In our previous study [43] in the absence of DNA-aneuploidy, a maximal CV of G0/1phase cells with a mean of $3.89 \%$ was empirically found. Although, in this study here CVs $\geqslant 10 \%$ were found in tumor cell-positive effusions and never in tumor cell-negative ones, we nevertheless no longer recommend $[43,44]$ using a $\mathrm{CV} \geqslant 10 \%$ as an indication of DNA-aneuploidy as it does not further increase the detection rate.

While most other authors applied DNA-flowcytometry, we currently prefer DNA-image-cytometry especially in cytologically equivocal effusions until multiparameter measurements allow the inclusion of immunological markers combined with high precision DAPI-DNA-measurements. Morphologic detection and separate measurement of only few abnormal cells ("rare events") are only possible in imagecytometry. These diagnostically important cells with increased DNA-content are mostly missed by single parameter DNA-flow-cytometry. Studies with a high rate of DNA-aneuploidy in cytologically equivocal effusions [20,21,42] also applied DNA-imagecytometry.

With improved precision of DNA-flow- and -imagecytometry and more sophisticated algorithms for histogram analysis, DNA-aneuploidy can be detected in a higher percentage of cytologically equivocal effusions than previously assumed. This fact should result in an increased sensitivity for the detection of malignant cells and thus decrease the number of cytologically equivocal effusions.

Our results demonstrate that, at this point, DNAimage-cytometry has a higher sensitivity for the detection of malignancy in effusions than DNA-flowcytometry. Both methods have a specificity of $100.0 \%$. As DNA-flow-cytometry applied to effusions is faster and less expensive to perform than -image-cytometry, the aim should be to improve this method, so that its sensitivity may be increased. In order to do so the rate of detection of DNA-aneuploidy in flow-cytometry, the often very small populations of tumor cells in effusions must be separated from overlaps by non-malignant cells.

Not only in DNA-image but also in -flow-cytometry, measurements for diagnostic purposes are strictly recommended only in combination with a cytological inspection of routine slides including quantitative evaluation of the ratio of the different cell types in each individual effusion. Furthermore, external diploid reference cells should be added in flow-cytometry in order to obtain an absolute calibration. This would also allow the detection of smaller deviations of DNA-stemlines (between 3-5\%) from the normal diploid value. 
The current advantages of DNA-image-cytometry are the following: the measurement may be performed on existing cytological routine slides; morphologically identifiable cells are individually measurable; remeasurements are possible at any time; a quality control of measured cells is possible on an "image gallery"; there is a relatively broad spectrum of indications (identification of malignant cells especially in borderline lesions and dysplasias, grading of tumor malignancy and monitoring of therapy); multiparametric measurements (combination with antigens); "rare event detections" (i.e., 9cEE) are possible and internal calibrations are possible.

The disadvantages of interactive DNA-imagecytometry are the following: cytodiagnostically trained personnel is necessary; only a limited number of cells are measurable (usually about 300); measurements are relatively time consuming (about $30 \mathrm{~min}$ ); preparation/staining is time consuming; the proportionality error of Feulgen-staining limits the precision of detection of aneuploid DNA-stemlines near 2c.

The general advantages of DNA-flow-cytometry are: it is easy to measure a representative number of cells (i.e. 20,000); the results are quickly available (10 min); a multiparametric analysis is possible (i.e., additional detection of cytoplasmatic antigens); there is no proportionality error of staining DNA by fluorescence dyes.

The disadvantages of DNA-flow-cytometry currently are: technically trained personnel is necessary; morphologically identifiable cell populations are not individually measurable; there is only a relatively small spectrum of indications (usually only grading of tumor malignancy); "rare event detections" are not possible (i.e., 9cEE); no repetition of measurements are possible; highly precise measurements of DNA using DAPI stain are only possible after enzymatic cell separation (loss of cytoplasm); separate probes are necessary (routine slides cannot be used); internal calibrations often reveal problems.

\section{Acknowledgements}

We wish to thank all clinicians who generously supported us by providing patient follow-up data. Furthermore, we would like to express our thanks to Mrs. B. Buckstegge and Mrs. H. Müschenborn for excellent preparation of slides.

\section{References}

[1] E.R. Banks, C.D. Jennings, S. Jacobs and D.D. Davey, Comparative assessment of DNA analysis in effusions by image analysis and flow cytometry, Diagn. Cytopathol. 10 (1994), 62-67.

[2] C.W.M. Bedrossian, Malignant Effusions. A Multimodal Approach to Cytologic Diagnosis, Igaku-Shoin Medical Publishers, New York, Tokyo, 1994.

[3] M. Bibbo, Comprehensive Cytopathology, WB Saunders Company, Philadelphia, 1991, pp. 541-614.

[4] S. Biesterfeld, K. Gerres, G. Fischer-Wein and A. Böcking, Polyploidy in non-neoplastic tissues, J. Clin. Pathol. 47 (1994), 38-42.

[5] A. Böcking, E. Striepecke, H. Auer and L. Füzesi, Static DNA cytometry: Biological background, technique and diagnostic interpretation, in: Compendium on the Computerized Cytology and Histology Laboratory, G.L. Wied, P.H. Bartes, D.L. Rosenthal and U. Schenck, eds, Tutorials of Cytology, Chicago, 1994, pp. 107-128.

[6] A. Böcking, DNA measurements: When and why?, in: Compendium on Quality Assurance, Proficiency Testing and Workload Limitations in Clinical Cytology, G.L. Wied, C.M. Keebler, D.L. Rosenthal, U. Schenck, T.M. Somrak and G.P. Vooijs, eds, Tutorials of Cytology, Chicago, 1995, pp. 170-188.

[7] A. Böcking, F. Giroud and A. Reith, Consensus report of the European Society for Analytical Cellular Pathology task force on standardization of diagnostic DNA-image cytometry, Analyt. Cell. Pathol. 8 (1995), 67-74.

[8] A. Böcking, Biologische Grundlagen und Anforderungen an die Methodik der statischen DNA-Zytometrie. Deutscher Ärzteverlag, herausgegeben von der Bundesärztekammer Köln 1997/98, Vol. 21, pp. 21-40.

[9] A. Böcking and N. Freudenberg, Standardisierte Befunderstellung in der extragenitalen Zytologie, Pathol. 19 (1998), 235236.

[10] A. Böcking, Standardisierung der zytopathologischen Diagnostik, Der Pathologe 19 (1998), 236-240.

[11] N. Böhm, E. Sprenger, G. Schlüter and W. Sandritter, Proportionalitätsfehler bei der Feulgen-Hydrolyse, Histochemie 15 (1968), 194-203.

[12] R. Chatélain, T. Schunck, E.M. Schindler, A.E. Schindler and A. Böcking, Diagnosis of prospective malignancy in koilocytic dysplasia of the cervix with DNA Cytometry, J. Reprod. Med. 34 (1989), 505-510.

[13] R. Chatélain, A. Willms, S. Biesterfeld, W. Auffermann and A. Böcking, Automated Feulgen staining with a temperature controlled staining machine, Analyt. Quant. Cytol. Histol. 11 (1989), 211-217.

[14] A.M. Croonen, P. Van der Valk, C.J. Herman and J. Lindeman, Cytology, immunopathology and flow cytometry in the diagnosis of pleural and peritoneal effusions, Lab. 58 (1988), 725732.

[15] P.N. Dean, C.D. Bagwell, T. Lindmo, R.F. Murphy and G.C. Salzman, Data file standard for flow cytometry, Analyt. Cytol. 11 (1990), 323-332. 
[16] F.R. De Castro, T. Molero, O. Acosta, G. Julia-Serda, J. Caminero, P. Cabrera and T. Carillo, Value of DNA analysis in addition to cytological testing in the diagnosis of malignant pleural effusions, Thorax 49 (1994), 692-694.

[17] A. Dejmek, C. Strömberg, B. Wiström and A. Hjerpe, Prognostic importance of the DNA ploidy pattern in malignant mesothelioma of the pleura, Analyt. Quant. Cytol. Histol. 14 (1992), 217-221.

[18] A.H. El-Habashi, S.M. Freeman, B. El-Morsi, M. El-Didi, A. Martin and A.J. Marrogi, DNA ploidy and proliferating cell nuclear antigen image analysis of peritoneal and pleural effusions, Acta Cytol. 41(3) (1997), 636-648.

[19] D.A. Evans, J.T. Thornthwaite, A.B.P. NG and E.V. Sugarbaker, DNA flow cytometry of pleural effusions, Analyt. Quant. Cytol. 5(1) (1983), 19-28.

[20] D.F. Fischler, S. Wongbunnate, D.A. Johnston and R.L. Katz, DNA content by image analysis. An accurate discriminator of malignancy in pericardial effusions, Analyt. Quant. Histol. 16(3) (1994), 167-173.

[21] S.C. Freni, J. James and F.J.A. Prop, Tumor diagnosis in pleural and ascitic effusions based on DNA Cytophotometry, Acta Cytol. 15(2) (1971), 154-162.

[22] H.F. Frierson, S.E. Mills and J.F. Legier, Flow-cytometric analysis of ploidy in immunohistochemically confirmed examples of malignant epithelial mesothelioma, Am. J. Clin. Pathol. 90 (1988), 240-243.

[23] F. Giroud, G. Haroske, A. Reith and A. Böcking, 1997 European Society for Analytical Cellular Pathology consensus report on diagnostic DNA image cytometry. Part II: Specific recommendations for quality assurance, Analyt. Cell. Pathol. 17 (1998), 201-208.

[24] W. Göhde, M. Us-Krasovec and A. Pogacnik, Die Bedeutung der Meßgenauigkeit bei der DNS-Zytophotometrie, Verh. Dtsch. Ges. Zyt. 19 (1995), 156-170.

[25] M.A. Hafiz, R.L. Becker, U.V. Mikel and G.F. Bah, Cytophotometric determination of DNA in mesotheliomas and reactive mesothelial cells, Analyt. Quant. Cytol. Histol. 10 (1988), 120 126.

[26] G. Haroske, W. Meyer, F. Theissig and K.D. Kunze, Increase of precision and accuracy of DNA cytometry by correcting diffraction and glare errors. Analyt. Cell. Pathol. 9 (1995), 1-12.

[27] G. Haroske, W. Meyer, F. Theissig and K.D. Kunze, DNA stemline and single-cell aneuploidy in static DNA cytometry: Statistical approaches, Analyt. Quant. Cytol. Histol. 18 (1996), 68.

[28] G. Haroske, V. Dimmer, W. Meyer and K.D. Kunze, DNA histogram interpretation based on statistical approaches, Analyt. Cell. Pathol. 15 (1997), 157-173.

[29] G. Haroske, F. Giroud, A. Reith and A. Böcking, ESACP consensus report on diagnostic DNA image cytometry. Part I, Basic considerations and recommendations for preparation, measurements and interpretation, Analyt. Cell. Pathol. 17 (1998), 189-200.

[30] D.W. Hedley, J. Philips, C.A. Rugg and I.W. Taylor, Measurement of DNA content as an adjunct to diagnostic cytology in malignant effusions, Eur. J. Cancer Clin. Oncol. 20(6) (1984), 749-752.
[31] T. Heiden, N. Wang and B. Tribukait, An improved Hedley method for preparation of paraffin-embedded tissues for flow cytometric analysis of ploidy and S-phase, Cytometry $\mathbf{1 2}$ (1991), 614-621.

[32] W. Hiddemann, R. Meister, D.B. von Bassewitz, B. Wörmann and T. Büchner, DNS-Aneuploidie: Ein hochspezifischer Marker für Malignität in der Diagnostik von Pleuraergüssen, Atemw. Lungenkrkh. 10 (1984), 328-330.

[33] W. Hiddemann, J. Schumann, M. Andreef, B. Barlogie, C.J. Hermann, R.C. Leif, B.H. Mayall, R.F. Murphy and A.A. Sandberg, Convention on nomenclature for DNA cytometry, Cancer Genet. Cytogenet. 13 (1984), 181-183.

[34] M.S.A. Huang, M.S. Tsai, T.H. Wang, M.S. Lin, I.W. Chong, K.L. Chen and J.J. Hwang, Flow cytometric DNA analysis of pleural effusions, Kaohsiung J. Med. Sci. 8 (1992), 640-646.

[35] M.S. Huang, M.S. Tsai, J.J. Hwang and T.H. Wang, Comparison of nucleolar organiser regions and DNA flow cytometry in the evaluation of pleural effusion, Thorax 49 (1994), 11521156.

[36] H. Isobe, K.S. Sridhar, R. Doria, F. Cohen, W.A. Raub, M. Saldana and A. Krishan, Prognostic significance of DNA aneuploidy in diffuse malignant mesothelioma, Cytometry 19 (1995), 86-91.

[37] M.A. Jones, S. Hitchcox, C.T. D'Ascanio, J. Papillo and H.M. Tarraua, Flow cytometric DNA analysis versus cytology in the evaluation of peritoneal fluids, Gynecologic Oncology $\mathbf{4 3}$ (1991), 226-232.

[38] M.G. Joseph, D. Banerjee, P. Harris, S. Gibson and R.G. McFadden, Multiparameter flow-cytometric DNA analysis of effusions: a prospective study of 36 cases compared with routine cytology and immunohistochemistry, Modern Pathol. 8(6) (1995), 686-693.

[39] L. Kapusta, I. Zbieranowski, C. Demianiuk and D. Murray, The role of DNA flow- and image cytometry in the evaluation of body cavity fluids, Analyt. Quant. Cytol. Histol. 13 (1991), 187-192.

[40] D. Kindermann and C.H. Hilgers, Glare-correction in DNA image cytometry, Analyt. Cell. Pathol. 6 (1994), 165-180.

[41] L.G. Koss, Diagnostic Cytology and Its Histopathologic Bases, 4th edn, JB Lippincott Company, Philadelphia, 1992.

[42] K.W. Matter-Walstra and R. Kraft, Atypical cells in effusions: Diagnostic value of cell image analysis combined with immunocytochemistry, Diagn. Cytopathol. 15 (1996), 263-269.

[43] H. Motherby, T. Marcy, M. Hecker, B. Roß, B. Nadjari, H. Auer, K.M. Müller, D. Häussinger, B.E. Strauer and A. Böcking, Static DNA cytometry as a diagnostic aid in effusion cytology. I. DNA aneuploidy for identification and differentiation of primary and secondary tumors of the serous membranes, Analyt. Quant. Cytol. Histol. 20(3) (1998), 153-161.

[44] H. Motherby, B. Nadjari, T. Remmerbach, T. Marcy, N. Pomjanskaja, W. Müller, K. Knops, D. Häussinger, B.E. Strauer and A. Böcking, Static DNA cytometry as a diagnostic aid in effusion cytology. II. DNA aneuploidy for identification of neoplastic cells in equivocal effusions, Analyt. Quant. Cytol. Histol. 20(3) (1998), 162-168.

[45] H. Motherby, B. Nadjari, P. Friegel, J. Kohaus, U. Ramp and A. Böcking, Diagnostic accuracy of effusion cytology, Diagn. Cytopathol. 20(6) (1998), 350-357. 
[46] H. Motherby, M. Kube, N. Friedrichs, B. Nadjari, K. Knops, A. Donner, B. Baschiera, P. Dalquen and A. Böcking, Immunocytochemistry and DNA-image cytometry in diagnostic effusion cytology. I. Prevalence of markers in tumorcell-positive and negative smears, Analyt. Cell. Pathol. 19 (1998), 7-20.

[47] H. Motherby, N. Friedrichs, M. Kube, B. Nadjari, K. Knops, A. Donner, B. Baschiera, P. Dalquen and A. Böcking, Immunocytochemistry and DNA-image cytometry in effusion cytology. II. Diagnostic accuracy in equivocal smears, Analyt. Cell. Pathol. 19 (1999), 59-66.

[48] H.W. Müller, A. Böcking and H. Auer, TV Cytometer CM 1 for computer aided tumor diagnosis, in: Compendium on the Computerized Cytology and Histology Laboratory, G.L. Wied, P.H. Bartels, D.L. Rosenthal and U. Schenck, eds, Chicago Tutorials of Cytology, 1994, pp. 376-387.

[49] M.G. Ormerod, B. Tribukait and W. Giaretti, Consensus report for the task force on standardisation of DNA flow cytometry in clinical pathology, Analyt. Cell. Pathol. 17 (1998), 103-110.

[50] W.L. Parry and G.P. Hemstreet, Cancer detection by quantitative fluorescence image analysis, J. Urol. 139 (1989), 270-274.

[51] M.M. Pinto, DNA analysis of malignant effusions, Analyt. Quant. Cytol. Histol. 14(3) (1992), 222-226.

[52] S. Pyrhönen, M. Tiainen, J. Rautonen, L. Tammilheto, A. Laasonen, K. Mattson and S. Knuutila, Comparison of DNA and karyotype ploidy in malignant mesothelioma, Cancer Genet. Cytogenet. 60 (1992), 8-13.

[53] L. Sànchez, M. Regh, S. Biesterfeld, R. Chatélain and A. Böcking, Performance of a TV image analysis system as a Microdensitometer, Analyt. Quant. Cytol. Histol. 12(4) (1990), 279 284.

[54] J. Schneller, E. Eppich, E. Greenebaum, F. Elequin, A. Sherman, R. Wersto and L.G. Koss, Flow cytometry and Feulgen cytophotometry in evaluation of effusions, Cancer $\mathbf{5 9}$ (1987), 1307-1313.

[55] A.I. Spriggs and M.M. Boddington, Atlas of serous fluid cytopathology. A guide to the cells of pleural, pericardial, peritoneal and hydrocele fluids, in: Current Histopathology Series, Vol. 14. G.A. Gresham, ed., Kluwer Academic Publishers, Dordrecht, 1989.

[56] K.J. Stonesifer, J. Xiang, N.A. Benson and R.C. Braylan, Flow cytometric analysis and cytopathology of body cavity fluids, Acta Cytol. 31 (1987), 125-130.

[57] F.B.J.M. Thunnissen, I.O. Ellis and U. Jütting, Quality assurance in DNA image analysis on diploid cells, Cytometry 27 (1997), 21-25. 


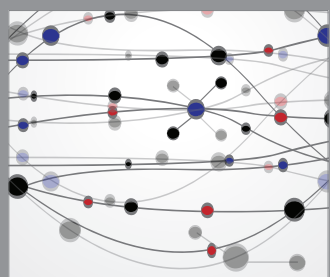

The Scientific World Journal
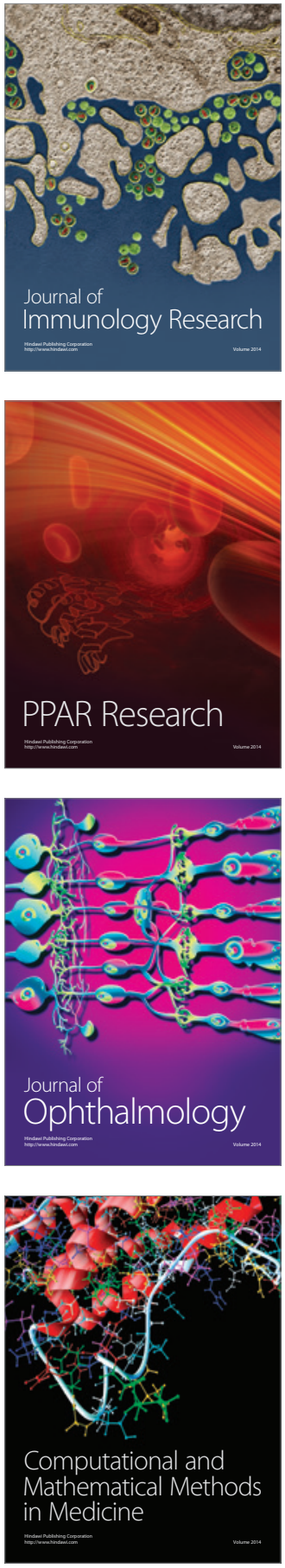

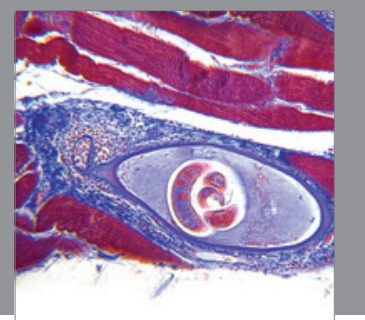

Gastroenterology

Research and Practice
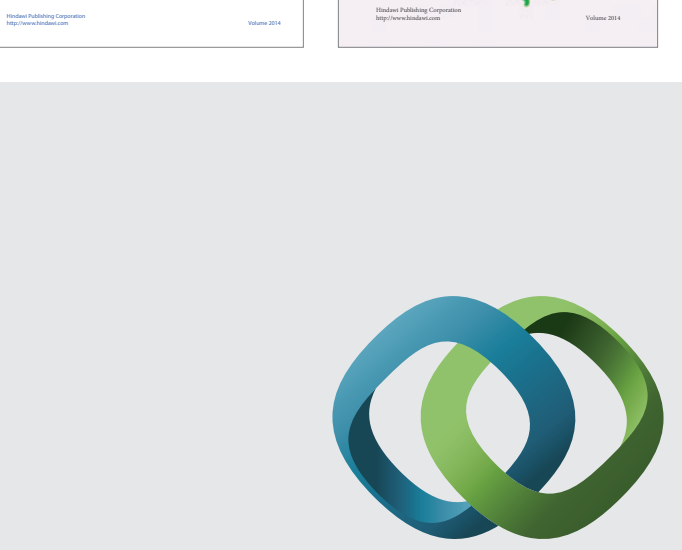

\section{Hindawi}

Submit your manuscripts at

http://www.hindawi.com
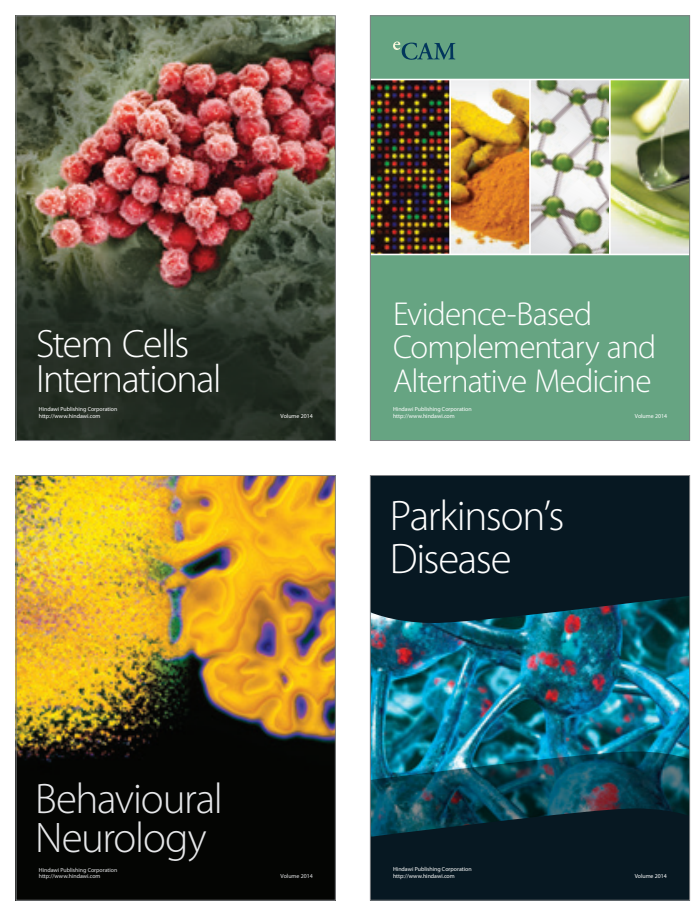

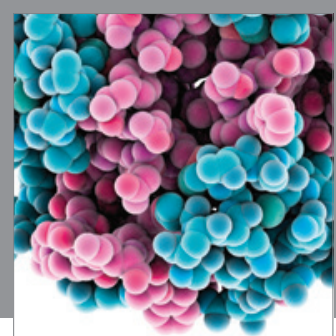

Journal of
Diabetes Research

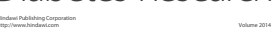

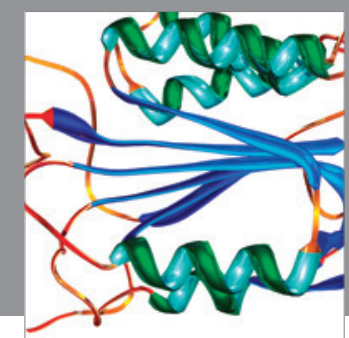

Disease Markers
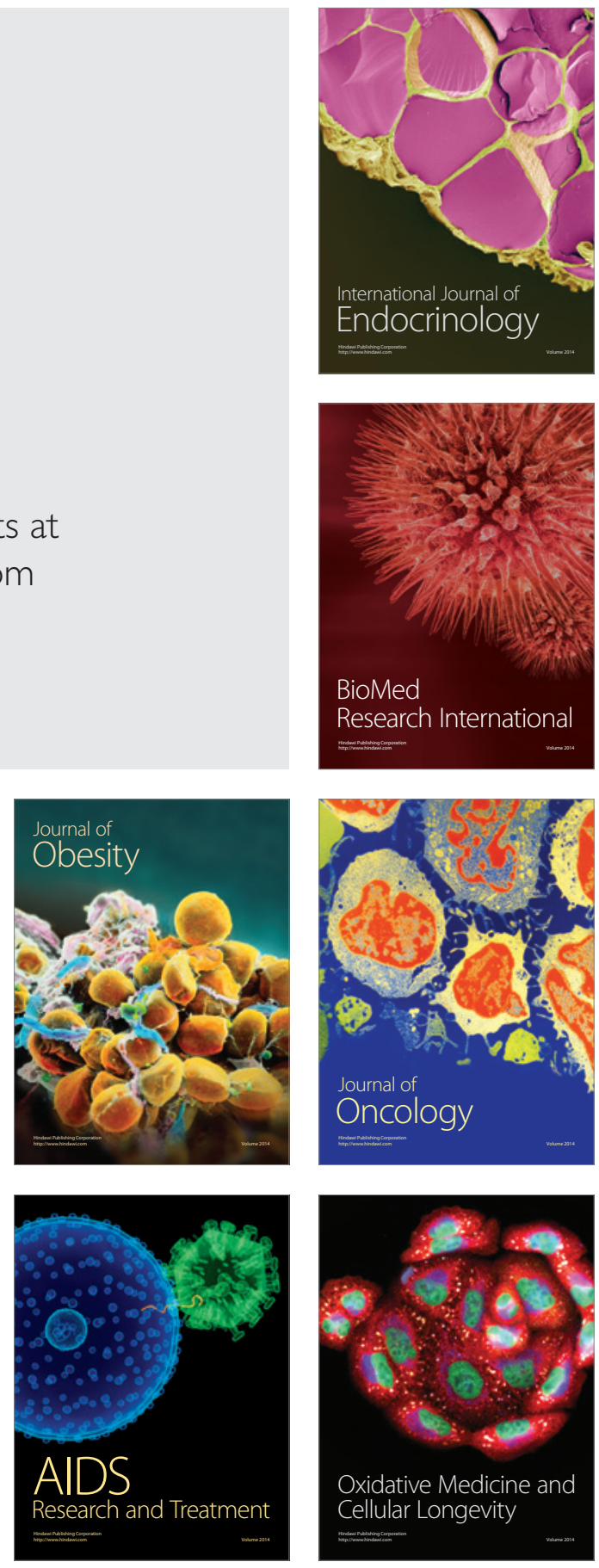